\title{
Molecular and cellular mechanisms of muscle aging and sarcopenia and effects of electrical stimulation in seniors
}

\author{
Laura Barberi (1) , Bianca Maria Scicchitano (2) $)^{\#}$, Antonio Musaro $(1,3)$ \\ (1) Institute Pasteur Cenci-Bolognetti, DAHFMO-unit of Histology and Medical Embryology, \\ IIM, Sapienza University of Rome, Italy: (2) Institute of Histology and Embryology, Catholic \\ University School of Medicine, Rome, Italy; (3) Center for Life Nano Science@Sapienza, \\ Istituto Italiano di Tecnologia, ItalyI \\ ${ }^{\#}$ These authors contributed equally to the work
}

\begin{abstract}
The prolongation of skeletal muscle strength in aging and neuromuscular disease has been the objective of numerous studies employing a variety of approaches. It is generally accepted that cumulative failure to repair damage related to an overall decrease in anabolic processes is a primary cause of functional impairment in muscle. The functional performance of skeletal muscle tissues declines during post- natal life and it is compromised in different diseases, due to an alteration in muscle fiber composition and an overall decrease in muscle integrity as fibrotic invasions replace functional contractile tissue. Characteristics of skeletal muscle aging and diseases include a conspicuous reduction in myofiber plasticity (due to the progressive loss of muscle mass and in particular of the most powerful fast fibers), alteration in muscle-specific transcriptional mechanisms, and muscle atrophy. An early decrease in protein synthetic rates is followed by a later increase in protein degradation, to affect biochemical, physiological, and morphological parameters of muscle fibers during the aging process. Alterations in regenerative pathways also compromise the functionality of muscle tissues. In this review we will give an overview of the work on molecular and cellular mechanisms of aging and sarcopenia and the effects of electrical stimulation in seniors.

Key Words: Sarcopenia, muscle atrophy, electrical stimulation, IGF-1, satellite cells, miRNA
\end{abstract}

Eur J Transl Myol - Basic Appl Myol 2015; 25 (4): xxx-yyy

It is generally accepted that cumulative failure to repair damage related to an overall decrease in anabolic processes is a primary cause of functional impairment in muscle aging. ${ }^{1-5}$

One of the important questions we should address when talking about aging and sarcopenia is, what happens to our muscle as we age? Skeletal muscle is an adaptive tissue of our organism. However, during aging there is a loss of the most powerful fast fibers. ${ }^{1,2}$ This muscle loss is replaced by fibrotic tissue that replaces the functional muscle, leading to muscle wasting.

Several mechanisms have been proposed to account for sarcopenia, which is a pathological state associated with aging and involves muscle atrophy (a decrease in the size of the muscle and muscle fibers) and a reduction in muscle tissue quality (Figure 1).

On a cellular level it has been demonstrated that one of the mechanisms associated with aging and sarcopenia is the reduction in the number of satellite cells and their proliferative activity. ${ }^{3}$ Satellite cells are normally activated during regeneration and play important role in muscle homeostasis. ${ }^{4}$ For decades, the age-related reduction in satellite cells number has been considered the critical limiting factors for muscle regeneration and repair. Nevertheless, even if a decline in the satellite cells might be associated with sarcopenia, the remaining and resident satellite cells should be sufficient to activate and sustain an adequate regenerative mechanism. However, the impaired regenerative potential of senescent muscle suggests a severe alteration in the functionality of satellite cells (Figure 2). ${ }^{5}$

We recently demonstrated that human satellite cells fail to differentiate when cultured in isochronic conditions. ${ }^{3}$ We analysed the ability of satellite cells derived from old subjects to differentiate when cultured in presence of either heterologous / heterochronic (from young donors) or autologous. Immunofluorescence analysis for the expression of $\mathrm{MyHC}$ revealed that aged satellite cells did not display 


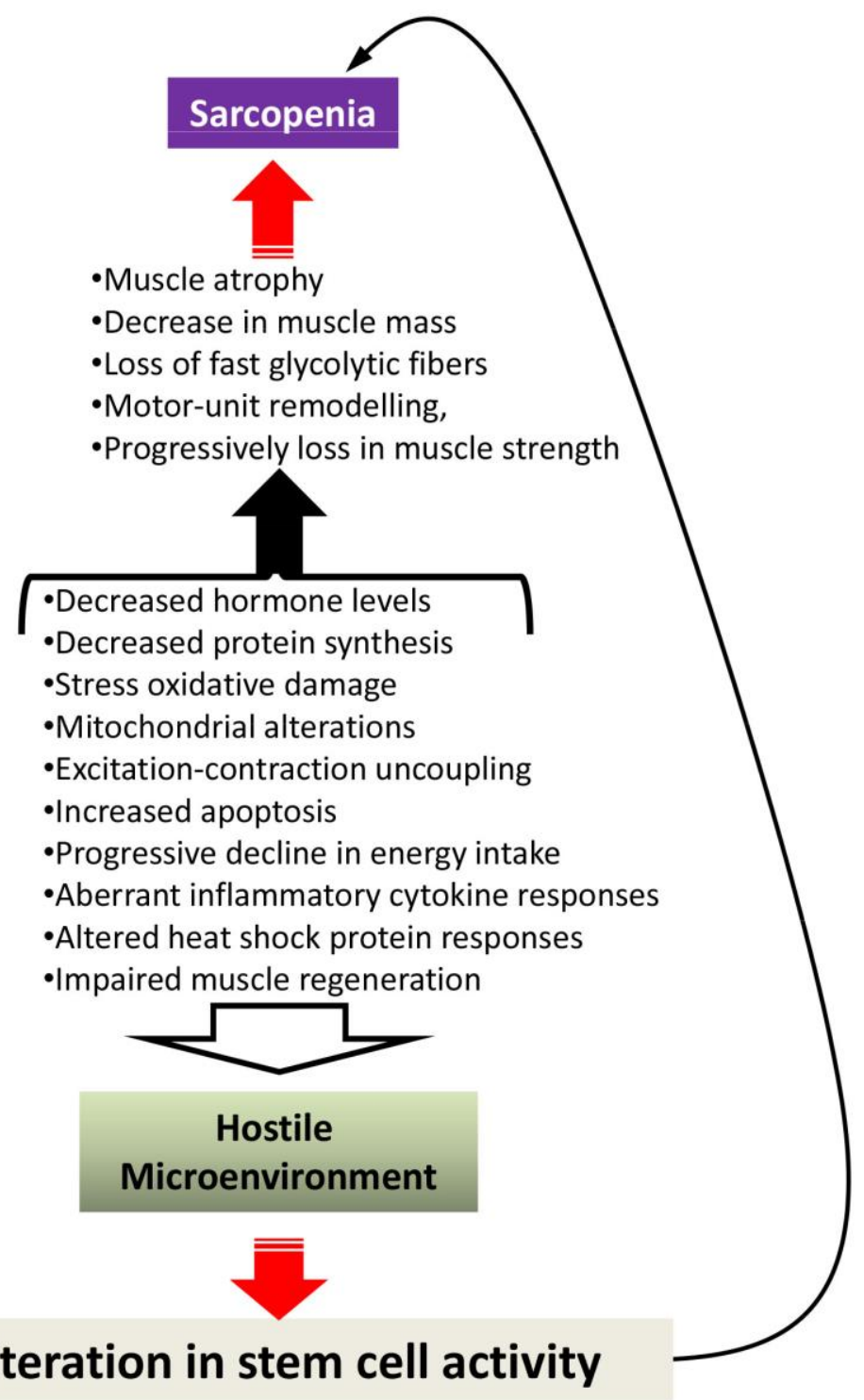

Fig 1. Schematic diagram of potential causes of sarcopenia. Current data point out that the development of muscle wasting is a multifactorial process and believed to be the result of both extrinsic factors, such as reduction in nutrition and exercise and intrinsic ones, involving changes in molecular and cellular levels. The factors responsible for the induction of sarcopenia might be also potential barriers for stem cell activity, creating an hostile environment that affects muscle regeneration and repair. On the other hand, alteration in muscle stem cell activity can contribute or exacerbate sarcopenia.

major defect in the propensity to fuse when differentiated under standard conditions, namely in DMEM supplemented with 5\% Horse serum. ${ }^{3}$ Of note, we observed that autologous serum (isochronic culture conditions) dramatically reduced muscle differentiation, which was partially rescued when aged satellite cells were differentiated in heterologous / heterochronic serum (from young donors). ${ }^{3}$ These results strongly supported the hypothesis that factors within the tissue itself in which muscle stem cells reside and operate are more important than the number of satellite cells and the source of the cells (i.e. from young or old donor) in determining the effectiveness of the regenerative response. ${ }^{6}$ In this context, one of the basic question to be addressed is: How to attenuate muscle atrophy and build a better muscle? There are different strategies. Physical exercise can be one option that can reverse sarcopenia. ${ }^{7,8}$

What is the effect of physical exercise? Both acute and prolonged resistance exercise stimulates the 


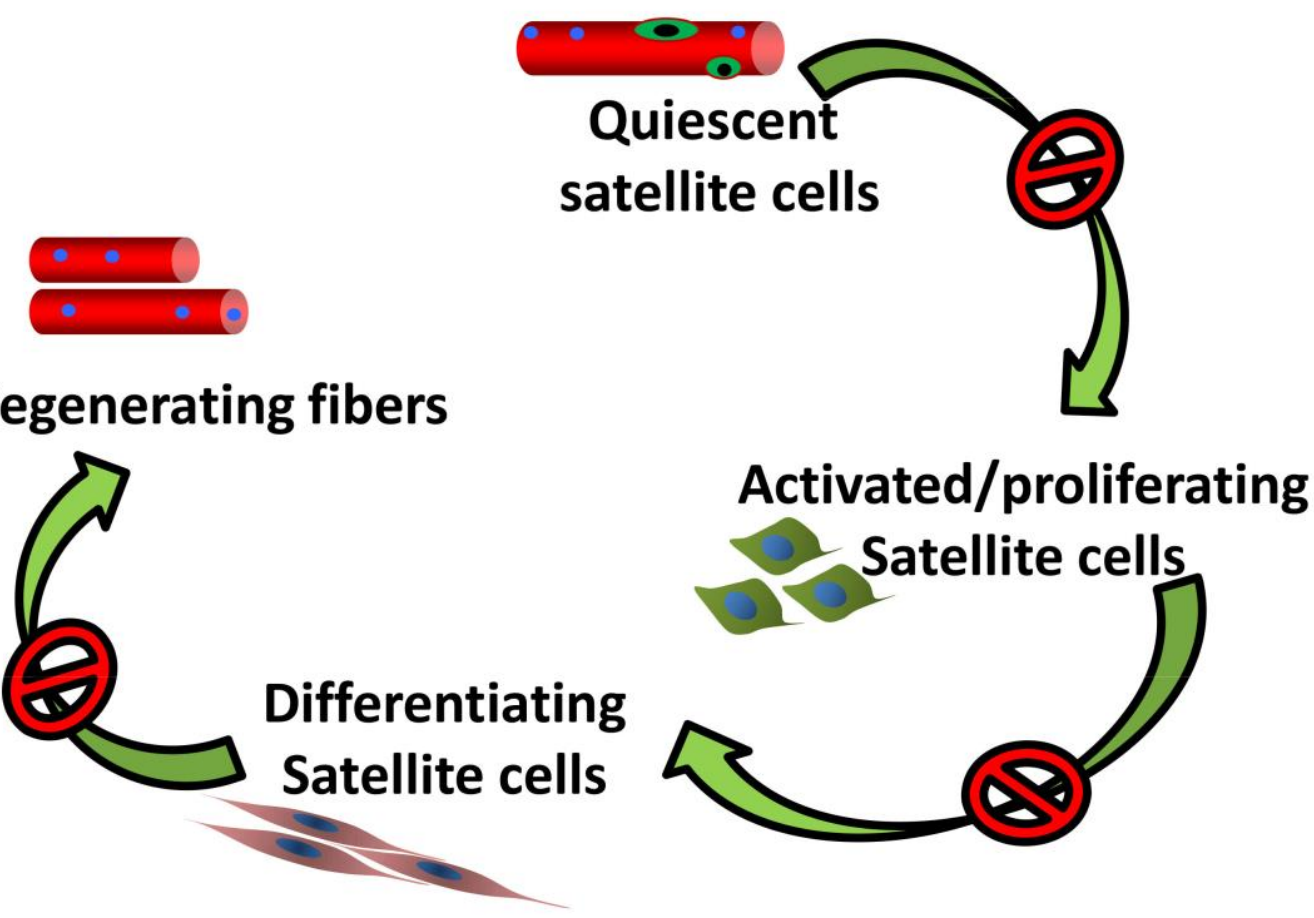

Fig 2. Schematic model outlining the stages of satellite cells: activation, entry into a proliferative state, differentiation, formation of new myofibers. The satellite cell activity may be impaired in the aging muscle

proliferation of satellite cells in healthy subjects. ${ }^{9-13}$ However, during aging it has been observed a reduced response of satellite cells to physical exercise. Interestingly, in humans, the reduced satellite cells proliferation with aging following exercise is associated with increased co-localization of myostatin, ${ }^{14}$ a negative regulator of muscle mass. ${ }^{15}$

It has been also demonstrated that autophagy is modulated by physical exercise and it plays important role in muscle homeostasis ${ }^{16}$. Human muscle biopsies from young, old sedentary and old sportsmen have been analyzed. It has been observed an increase in the autophagy in the muscle of people that practice sports, ${ }^{9}$ suggesting that exercise in some way activate an important system that detoxifies the cells from accumulation of toxic aggregates. Another factor that is associated with physical exercise is Insulin-like growth factor 1 (IGF-1). ${ }^{17}$ It has been demonstrated that IGF-1 increases after 5-10 min of moderate- to high-intensity exercise ${ }^{18-22}$ and that skeletal muscle is a source for the production of IGF-1 following exercise. $^{23}$ The IGF-1 gene gives rise to a heterogeneous pool of mRNA transcripts 2 , which are the results of several events (or combination of these events), including use of alternative transcription start sites located in leader exons (exons 1 and 2); alternative post-transcriptional exon splicing; and use of different polyadenylation site. These multiple IGF-1 mRNAs transcripts encode different isoforms of IGF-1 precursor peptide, which undergo post- translational cleavage to release the biologically active mature (70 amino acid long) IGF-1. ${ }^{2}$ In humans, three different IGF-1 isoforms have been identified: the IGF- 1Ea, IGF-1Eb and IGF-1Ec. ${ }^{24}$ The predominant IGF-1 mRNA variant expressed in resting skeletal muscle encodes for IGF-1Ea isoform, whereas in muscles subjected to damage or exercise the IGF-1Ec splice variant is upregulated. We also demonstrated that vibratory-proprioceptive training was accompanied by a selective two- fold increase in IGF-1Ec mRNA variants. This was associated to with a $10 \%$ increase of myofiber diameter of the fast twitch fibers and muscle strength. ${ }^{25}$.In another work, it has been demonstrated that viral expression of IGF-1 enhanced resistance exercise- induced hypertrophy and increased in contractile functions as well as preventing atrophy during detraining of 12 weeks. ${ }^{26}$ Interestingly, it has been observed that IGF-1 induced muscle hypertrophy via a combination of satellite cell activation and increasing protein synthesis in differentiated myofibers. ${ }^{27}$ IGF-1 is an anabolic factor that plays important role in tissue homeostasis. Several years ago we generated a transgenic mouse in which the local isoform of IGF-1 (mIGF-1) is driven by MLC 


\section{Molecular and cellular mechanisms of muscle aging and effects of FES}

Eur J Transl Myol - Basic Appl Myol 2015; 25 (4): 231-236

promoter (MLC/mIGF-1). ${ }^{28}$ Transgenic animals exhibits marked skeletal muscle hypertrophy with no undesirable side effects such as tumor formation. ${ }^{28}$ The increased muscle mass in mIGF-1 transgenic mice was associated with augmented force generation compared to age-matched wild type littermates. ${ }^{28}$ Examination of two year-old animals revealed that whereas wild type mice underwent characteristic muscle atrophy, expression of the mIGF-1 transgene was protective against normal loss of muscle mass during senescence. $^{28}$ Over-expression of the mIGF-1 transgene also preserved the regenerative capacity of senescent muscle tissues stimulating both the activity of satellite cells and the recruitment of circulating stem cells. $^{29}$

Interestingly, skeletal muscle has been considered as an endocrine organ that produces and releases cytokines, known as myokines, in response to contraction, influencing metabolism in other tissues and organs (reviewed in Pedersen et al). ${ }^{30}$ Among these, IL- 6 is produced by skeletal muscle in response to intense, prolonged exercise and it regulates carbohydrate and lipid metabolism, and satellite cell proliferation. ${ }^{31,32}$ Nevertheless, IL-6 can exert also catabolic activity, interfering with IGF-1 signaling. ${ }^{32,33}$ The complex actions of IL-6 may be linked to the different manners by which this cytokine signals at the plasma membrane and by the different signaling pathways that can activate. ${ }^{31,32}$. Based on the activation of either classic or trans-signaling, IL-6 can promote markedly different cellular responses. IL-6 transsignaling, which require the soluble IL6R (sIL6R), is pro-inflammatory, whereas classic IL-6 signaling, mediated by membrane-bound receptor, promotes regenerative or anti-inflammatory activities of the cytokine. $^{34}$ It is plausible that IL-6 blocks IGF-1 activity in skeletal muscle cells where IL-6 levels is high and IGF-1 levels is low (i.e. ageing conditions) through blocking its downstream signalling cascades.

These evidences suggest that training and regular exercise, by increasing functional autophagy, myokines and IGF-1 expression, can attenuate the pathological signs of sarcopenia, while increasing muscle strength and decreasing fall risk.

Nevertheless, one of the problems associated with aging is that some people cannot move because of pathological conditions like pain, osteoarthritis and so on. Is there an alternative approach instead of physical exercise for these people? Helmut Kern designed a specific way of analyzing the electrical stimulation ${ }^{35}$ and to address the question: can electrical stimulation mimic the effect of physical exercise?

In particular, a stimulator for neuromuscular electrical stimulation was designed, especially suiting the requirements of elderly people with diminished fine motor skills. ${ }^{35}$ As detailed in Kern et al., ${ }^{36}$ subjects were exposed to regular neuromuscular ES training (swelling current) for a period of 9 weeks, starting two times a week for the first 3 weeks and then switching to three times a week for the next 6 weeks, amounting to a total of 24 training sessions ( $3 \times 10$ minutes each session). Electrical stimulation was performed with a two channel custom-built battery-powered stimulator $^{36}$ The subjects applied two conductive rubber electrodes ( $\left.9 \times 14 \mathrm{~cm} ; 126 \mathrm{~cm}^{2}\right)$ which were attached to the skin by wet sponge on the anterior thigh on both sides (left/right). The electrode pairs for left and right thigh were connected to the two channels of the stimulator. This allowed independent activation of the left and right thigh muscles, which were stimulated in an alternative manner. Each repetition (i.e. ES evoked muscle contraction) was evoked by a $3.5 \mathrm{~s}$ train $(60 \mathrm{~Hz})$ of electrical pulses (rectangular, biphasic, width $0.6 \mathrm{~ms}$ ). Consecutive contractions of the same thigh were separated by $4.5 \mathrm{~s}$ off intervals. In this study constant voltage stimulation devices were applied. The subjects were instructed to increase the stimulation intensity until their maximum sensory tolerance level was reached. With this intensity all of the subjects achieved full knee extension. Nevertheless, the applied current and voltage was recorded by the stimulation device for each training session. The mean stimulation current was $128 \pm 16 \mathrm{~mA}$ and voltage of $39 \pm 14 \mathrm{~V}$. Of note, none of the trained subjects declared problematic events during training sessions; they reported slight pain clinically not relevant at rest before ES, without changes through ES training. ${ }^{36}$ What the group of Kern's collaborators demonstrated is that electrical stimulation did improve muscle performance. ${ }^{36}$ The increase in muscle strength, was associated with an increase of muscle fibers and most importantly with an increase of fast fibers, which are related to the power of the skeletal muscle. ${ }^{36} \mathrm{We}$ asked: what is the mechanism associated with this increase of muscle strength and increase in muscle mass?

Since IGF-1 is one of the factors that are activated during physical exercise, we verified whether electrical stimulation was able to induce an increase in IGF-1 expression. At first, we analyzed the expression of the different types (isoforms) of IGF-1. All of them were up regulated after electrical stimulation. ${ }^{36}$ Then we analyze some downstream pathways activated by IGF1. We demonstrated that electrical stimulation stimulates not only anabolic pathways, but negatively modulates muscle catabolism. ${ }^{36}$ Another component that we analyze is the collagen expression. There is remodeling, not only during physical exercise but also in electrical stimulation of extra cellular matrix (ECM). We observed an up regulation of the three different forms of collagen (I, III and VI) in electrical stimulated muscle. ${ }^{36} \mathrm{We}$ then verified whether the increase in collagen stimulates fibrosis. Of note histological did not reveal any accumulation of fibrotic tissue in electrical stimulated muscles. ${ }^{36}$ To further support the morphological evidences, we analyzed one of the important controllers of fibrosis, namely miR29. ${ }^{37}$ The 


\section{Molecular and cellular mechanisms of muscle aging and effects of FES}

Eur J Transl Myol - Basic Appl Myol 2015; 25 (4): 231-236

electrical stimulation regulates miR29, which might block the accumulation of fibrosis. ${ }^{36}$ We then analyzed the number of satellite cells that can be activated by electrical stimulation. We wanted to verify whether electrical stimulation, similarly to exercise, can increase the activity of these cells. Electrical stimulation indeed increased the number of satellite cells. $^{36}$ Moreover, we analyzed other molecular markers normally associated with the commitment of satellite cells: Myogenin, miR-206 and miR-1. Electrical stimulation increased these molecular markers. $^{36}$

In conclusion, what we demonstrated is that electrical stimulation, which can be applied to people that cannot carry out normal physical activity, modulates similar factors associated with physical exercise. In particular, electrical stimulation activates IGF-1. IGF-1, once stimulated activates an anabolic pathway increasing protein synthesis while reducing protein degradation and activating satellite cells. This all leads to an increased muscle performance. The results collected here suggest that electrical stimulation, similarly to physical exercise, attenuate the functional decline associated with aging, improving muscle strength and mass, maintaining the overall size of muscle fibers (decreasing during aging), activating satellite cells and guaranteeing muscle adaption. We can also speculate that electrical stimulation mimics the effect of endurance training, based on the evidences that it does not induce muscle damage, and activates a comparable molecular network to endurance training. All of these data might help to design therapeutic strategies to counteract muscle atrophy associated with aging.

\section{Acknowledgement}

The presentation of this work is the results of a collaboration among different groups coordinated by Helmut Kern (Institute of Physical Medicine and Rehabilitation and Ludwig Boltzmann Institute of Electrical Stimulation, Vienna, Austria).

Work in the authors' laboratories has been supported by Telethon, ASI, PRIN, and AFM. This study was also supported by Cross Border Cooperation Programme SLOVAKIA-AUSTRIA (Interreg-IVa).

\section{Authors contribution}

All the authors drafted the article and revised it critically. The authors do not have financial interest in relation to this submission

\section{Corresponding Author}

Antonio Musarò, Unit of Histology and Medical Embryology, Via A. Scarpa 14, Rome 00161, Italy

E-mail: antonio.musaro@uniroma1.it

E-mails of Co-Authors:

Laura Barberi: laura.barberi@uniroma1.it

Bianca Maria Scicchitano:

biancamaria.scicchitano@rm.unicatt.it

\section{References}

1. Vinciguerra M, Musaro A, RosenthalN. Regulation of muscle atrophy in aging and disease. Adv Exp Med Biol. 2010; 694:211-33.

2. Scicchitano BM, Rizzuto E, Musarò A. Counteracting muscle wasting in aging and neuromuscular diseases: the critical role of IGF-1. Aging 2009; 451-7.

3. Barberi L, Scicchitano BM, De Rossi M, et al. Age-dependent alteration in muscle regeneration: the critical role of tissue niche. Biogerontology. 2013; 14:273-92

4. Musarò A. The Basis of Muscle Regeneration. Advances in Biology 2014, 2014: 1-16.

5. Carosio S, Berardinelli MG, Aucello M, Musarò A. Impact of ageing on muscle cell regeneration. Ageing Res. Rev. 2011; 10:35-42.

6. Conboy IM, Conboy MJ, Wagers AJ, et al. Rejuvenation of aged progenitor cells by exposure to a young systemic environment. Nature 2005; 433: 760-4.

7. Snijders T, Verdijk LB, van Loon LJ. The impact of sarcopenia and exercise training on skeletal muscle satellite cells. Ageing Res. Rev. 2009; 8: 328-38.

8. Mosole S, Carraro U, Kern H, et al. Long-term high-level exercise promotes muscle reinnervation with age. $\mathbf{J}$ Neuropathol Exp Neurol. 2014; 73:284-94.

9. Kadi F, Schjerling P, Andersen LL. et al. The effects of heavy resistance training and detraining on satellite cells in human skeletal muscles. The Journal of Physiology 2004; 558: 1005-12.

10. Mackey AL, Holm L, Reitelseder S. et al. Myogenic response of human skeletal muscle to 12 weeks of resistance training at light loading intensity. Scandinavian journal of medicine \& science in sports 2010; 21: 773-82.

11. Mikkelsen UR, Langberg H, Helmark IC. et al. Local NSAID infusion inhibits satellite cell proliferation in human skeletal muscle after eccentric exercise. Journal of applied physiology 2009; 107: 1600-11.

12. Farup J, Rahbek SK, Riis S. et al. Influence of exercise contraction mode and protein supplementation on human skeletal muscle satellite cell content and muscle fiber growth. Journal of applied physiology (Bethesda, Md : 1985) 2014; 117: 898-909.

13. 13 Farup J, Rahbek SK, Knudsen IS. et al. Whey protein supplementation accelerates satellite cell proliferation during recovery from eccentric exercise. Amino Acids 2014; 46: 2503-16.

14. McKay BR, Ogborn DI, Bellamy LM. et al. Myostatin is associated with agerelated human muscle stem cell dysfunction. FASEB J. 2012; 26: 2509-21. 


\section{Molecular and cellular mechanisms of muscle aging and effects of FES}

Eur J Transl Myol - Basic Appl Myol 2015; 25 (4): 231-236

15. McPherron AC, Lawler AM, Lee SJ. Regulation of skeletal muscle mass in mice by a new TGFbeta superfamily member. Nature. 1997; 387:8390.

16. Carnio S, LoVerso F, Baraibar MA, Autophagy impairment in muscle induces neuromuscular junction degeneration and precocious aging. Cell Rep. 2014; 8:1509-21.

17. Adamo ML, Farrar RP. Resistance training, and IGF involvement in the maintenance of muscle mass during the aging process. Ageing Res Rev. 2006; 5:310-31. Epub 2006 Sep 1.

18. Cappon J, Brasel JA, Mohan S, Cooper DM. Effect of brief exercise on circulating insulinlike growth factor I. J Appl Physiol 1994;76: 2490-6.

19. Schwarz AJ, Brasel JA, Hintz RL. et al. Acute effect of brief low- and high- intensity exercise on circulating insulinlike growth factor (IGF) I, II, and IGF-binding protein-3 and its proteolysis in young healthy men. J Clin Endocrinol Metab 1996; 81:3492-7.

20. Hornum M, Cooper DM, Brasel JA. et al. Exercise-induced changes in circulating growth factors with cyclic variation in plasma estradiol in women. J Appl Physiol. 1997; 82:1946-51.

21. Wallace JD, Cuneo RC, Baxter R. et al. Responses of the growth hormone $(\mathrm{GH})$ and insulin-like growth factor axis to exercise, $\mathrm{GH}$ administration, and $\mathrm{GH}$ withdrawal in trained adult males: A potential test for $\mathrm{GH}$ abuse in sport. J Clin Endocrinol Metab. 1999; 84:3591601.

22. Kostka T, Patricot MC, Mathian B. et al. Anabolic and catabolic hormonal responses to experimental two-set low- volume resistance exercise in sedentary and active elderly people. Aging Clin Exp Res 2003; 15:123-30.

23. Berg U, Bang P. Exercise and circulating insulinlike growth factor I. Horm Res. 2004; 62: 50-8.

24. Sandri M, Barberi L, Bijlsma AY. et al. Signalling pathways regulating muscle mass in ageing skeletal muscle: the role of the IGF1-AktmTOR-FoxO pathway. Biogerontology. 2013; 14:303-23.

25. Kern H, Pelosi L, Coletto L. et al. Atrophy/hypertrophy cell signaling in muscles of young athletes trained with vibrationalproprioceptive stimulation. Neurol Res. 2011; 33:998-1009.
26. Lee S, Barton ER, Sweeney HL, Farrar RP. Viral expression of insulin- like growth factor-I enhances muscle hypertrophy in resistancetrained rats. J Appl Physiol (1985). 2004; 96:1097- 104.

27. Barton-Davis ER, Shoturma DI, Sweeney HL. Contribution of satellite cells to IGF-I induced hypertrophy of skeletal muscle. Acta Physiol Scand. 1999; 167:301-5.

28. Musarò A, McCullagh K, Paul A. et al. Localized Igf-1 transgene expression sustains hypertrophy and regeneration in senescent skeletal muscle. Nature Genetics 2001; 27: 195-200.

29. Musarò A, Giacinti C, Borsellino G. et al. Muscle restricted expression of $\mathrm{mIGF}-1$ enhances the recruitment of stem cells during muscle regeneration. Proc Natl Acad Sci U S A 2004; 101: 1206-10

30. Pedersen BK, Akerström TC, Nielsen AR, Fischer CP. Role of myokines in exercise and metabolism. J Appl Physiol (1985). 2007; 103:1093-8.

31. Fuster JJ, Walsh K,. The good, the bad, and the ugly of interleukin-6 signaling. EMBO J. 2014; 33: 1425- 7.

32. Munoz-Canoves P, Scheele C, Pedersen BK, Serrano AL. Interleukin-6 myokine signaling in skeletal muscle: a double-edged sword? FEBS J. 2013; 280: 4131-48.

33. Pelosi L, Berardinelli MG, De Pasquale L. Functional and Morphological Improvement of Dystrophic Muscle by Interleukin 6 Receptor Blockade. EBioMedicine 2015; 2: 274-5

34. Rose-John, S. IL-6 trans-signaling via the soluble IL-6 receptor: importance for the proinflammatory activities of IL- 6. Int.J Biol.Sci. 2012; 8:1237-47.

35. Krenn M. Haller M. Bijak M. et al. Safe neuromuscular electrical stimulator designed for the elderly. Artif. Organs 2011; 35:253-6.

36. Kern H, Barberi L, Löfler S. et al. Electrical stimulation counteracts muscle decline in seniors. Front Aging Neurosci. 2014; 6:189.

37. He Y, Huang C, Lin X, Li J. MicroRNA-29 family, a crucial theraeutic target for fibrosis diseases. Biochimie 2013; 95:1355-1359. 